\title{
Research on Pricing Models and Strategies of Online Group- Buying
}

\author{
Qiuhua Ding, ${ }^{1, *}$, Shixin Zhang ${ }^{2}$ \\ ${ }^{1}$ Qiuhua Ding, ${ }^{2}$ Shixin Zhang, Lanzhou University of Technology, 730050 Lanzhou, Gansu, China
}

\begin{abstract}
In recent years, the Online Group-Buying has entered a state of development in full swing. The particularity of the OGB platform compared with the traditional market determines that its marketing and price setting model must be different from the traditional market. Based on the summary of domestic and foreign scholars' literature on the pricing and strategy research of group buying business models, this article analyzes various factors affecting group buying prices. Under various assumptions, parameters and variables, through mathematical calculation and analysis, the optimal price and maximum profit for the healthy development of the enterprise are obtained, and how the merchants on the group buying website should adopt corresponding price-setting strategies and profit models to made some suggestions.
\end{abstract}

\section{Introduction}

As a new shopping mode, OGB has become a new choice for people's daily consumption. If merchants want to attract customers and make profits through group buying, they must set up appropriate booking group buying prices and prices for traditional buying methods. They also need to pay attention to the conditions under which booking group buying can obtain higher profits than ordinary sales. In this context, this article considers the pricing of products under the sales model where the merchants organize group purchases on third-party online shopping platforms from the perspective of merchants, hoping to help merchants make appropriate pricing plans. The groupbuying model originated in foreign markets. Webster et al. (1968) studied the group-buying model in the United States and believed that participants in the group would jointly bear the risk for the purchase of the same product[1]. Kauffman (2002) analyzed the connotation of the group buying model in a more in-depth manner. He believed that OGB can allow consumers to accumulate, so that they have the ability to negotiate prices with merchants, and finally get products at lower prices[2]. Tang Fangcheng et al. (2013) analyzed pricing models in two markets, monopoly and perfect competition, based on the bilateral market theory[3]. Li Guohao et al. (2013) considered the game of two retailers, and contained two factors of service and price, and then solved the game model, and studied the two cases where there is or not symmetry between the two retailers[[4]. How should the two retailers set up their own sales strategies and prices?

According to the analysis report of OGB industry of China report network, with the improvement of Internet penetration, the number of online group buying in China is also increasing year by year. In 2016, the number of OGB users in China was 0.97 million, and in 2020, the number of online group buying users has increased to 4.74 million.It can be seen that China's online group buying market has a good development prospect and a large demand for users,Therefore, it is necessary to study the pricing model and strategy of group purchase on the Internet,See Figure 1 for details.

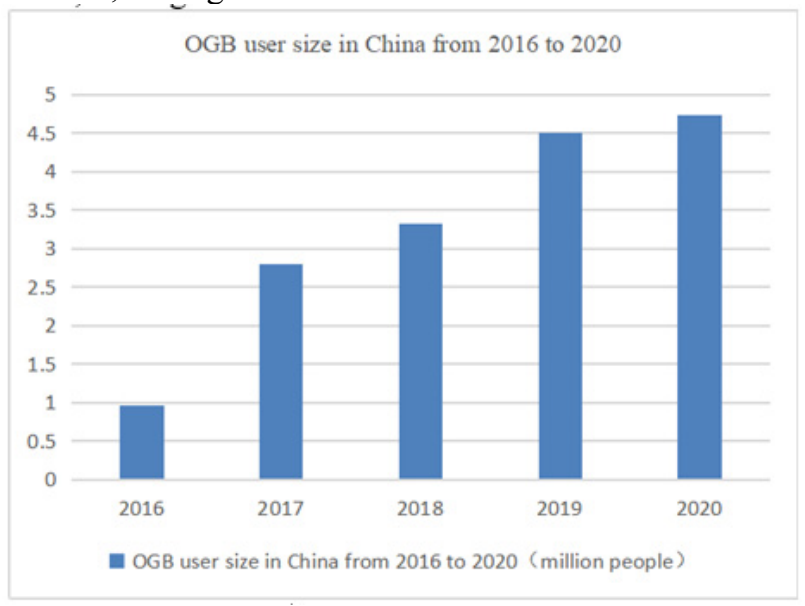

Source: China report network

Fig. 1. 2016-2020 OGB user size in China

\section{Research on the price and strategy of Online Group-buying}

\subsection{Factors influencing Online Group-buying pricing}

\subsubsection{Market structure}

Ju Chunhua et al. (2006) analyzed and compared various modes of group buying, and pointed out that group buying directly and actively organized by retailers is better than 
the franchise in terms of technology, product structure, information acquisition and delivery, etc. Group buy[5]. Similar to traditional product pricing, the structure of the OGB market will also affect the pricing of products by merchants. In order to win customers in the competition, for consumers, the prices offered by merchants are relatively low, and even to compete for users, they may compete with each other to reduce prices. At the same time, the group buying platform will also give consumers certain subsidies. Both parties have invested heavily in price subsidies to increase the number of users, and continue to use this method to cultivate user stickiness.

\subsubsection{Commission rate}

Chen Haitao et al. (2015) use a game model to believe that the group buying model can achieve a win-win situation between merchants and consumers, and both will achieve greater benefits[6]. At present, OGB platforms achieve their own profits by drawing commissions, and the commission ratio drawn is also one of the important factors that affect the price set by merchants. In this model, a reasonable commission ratio is not only helpful for merchants to formulate sales strategies, attract more consumers, and maximize the profits of each supply chain entity in group buying, thereby promoting a virtuous circle and development of the group buying supply chain. , It also has a certain impact on merchants' group-buying pricing decisions.

\subsubsection{Product portfolio}

Because customers have different consumer preferences, providing a diversified choice of goods will have a certain impact on the consumer base. Therefore, many groupbuying merchants control the scale of group-buying by restricting the selectivity of the goods distributed. However, the limited scale of group buying reduces consumer surplus. For physical products, consumers have different needs, because different people have different preferences for quality, appearance, or LOGO image or function, and the budget constraints of each consumer are also uneven.

In addition, the price demand elasticity of the consumers participating in the group is relatively strong, so there is a considerable part of low-value consumers, they may participate in the group and reduce the price so that all participants can gain benefits. At this time, the product mix should consider the differences in consumer demand rather than the number of purchases. Differentiating charges for the same batch of consumers affects all consumers by attracting more participants. For group buying of combined products, because some products are substitutes, the growth rate of the total value of the products is diminishing, which means that the total value of the combined product is less than the simple addition of the value of each individual product, so it should be based on the total value Work out the corresponding price strategy.

\subsubsection{Whether the user is single-homed or multi- homed}

Single-homing means that a user establishes contact with only one platform, and multi-homing means that a user establishes contact with multiple platforms. If users on both sides of the platform are single-homed, users on both sides of the platform can enjoy cheaper prices. If one side is single-homed and the other side is multi-homed users, the platform will set high prices for multi-homed users to subsidize single-homed users. The user only registers on a certain group buying website, then the user is singlehomed; otherwise, it is multi-homed. In order to meet their own diversified consumer needs, users often register on multiple websites to obtain the greatest discount. For merchants, they also seek to reach as many cooperative relationships as possible with various group buying websites, promote and sell their own products as much as possible, and increase business volume. Therefore, whether it is a consumer or a merchant, it is more common for users to have multi-homing. In the case of multihoming, the pricing strategy of merchants is more complicated. This article will not conduct in-depth research.

\subsection{Parameter settings and assumptions}

The function of $D=A-B P$ is to represent the base of demand for this kind of commodity in the market. $A$ Indicates the elasticity of the market, $B$ Indicates the elasticity of the market. $P$ Indicates the price at which the product is sold when the merchant organizes the group purchase; $P_{1}$ represents the product's booking group purchase price; $C$ represents the merchant's unit cost; $\lambda$ represents the proportion of customers who participate in the group purchase from the product who booked the group purchase and $0 \leqslant \lambda<1 ; \theta$ indicates the proportion of consumers who are additionally attracted because the booking group purchase price is lower than the normal price of the product, and $0<\theta \leqslant 1$; T indicates the minimum number of people who book the group purchase specified by the merchant; $\mu$ indicates the success of the booking group purchase Rate, $0 \leqslant \mu \leqslant 1$.

The following are assumptions:

Hypothesis 1: Regardless of the pre-booked group purchase price of the product in the market, there will always be certain consumers who do not participate in the group and directly purchase the product. This part of customers is unwilling to wait for the waiting period before the group starts or the preferential price for group purchases. Not sensitive.

Hypothesis 2: Customers who decide to book a group purchase will pay a certain amount to the merchant. If the group is successful, the part of the customer needs to pay the balance; if the group fails, the merchant will refund the prepayment and will not consider the refund. cost of.

Hypothesis 3: The customer's sensitivity to price is greater than the attractiveness of booking group buying to customers, that is $\theta \geqslant \lambda$. This ensures that the merchants organize booking group buying to expand demand, 
otherwise booking group buying will be meaningless.

\subsection{Model establishment and analysis}

\subsubsection{Merchant's pricing decision when not organizing scheduled group buying activities}

If the merchant does not organize booking group purchases, but just sells directly, then the market demand is $D_{0}=A-B P_{0}$, The price of a unit commodity is $P_{0}$, and the profit function of the merchant when selling is expressed as:

$$
\pi_{0}=\left(P_{0}-C\right)\left(A-B P_{0}\right)
$$

According to formula (1), $\pi_{0}$ is a quadratic function of $P_{0}$, so it has an optimal value:

$$
\frac{\partial \pi_{0}}{\partial P_{0}}=-2 B P_{0}+A+B C
$$

Because the first-order necessary conditions can find the best price and the best supplier income when directly selling products.

$$
\begin{aligned}
& \text { Best selling price: } \quad P_{0}{ }^{*}=\frac{A+B C}{2 B} \\
& \text { Supplier total revenue: } \pi_{0}{ }^{*}=\frac{(A-B C)^{2}}{4 B}
\end{aligned}
$$

\subsubsection{Merchant's pricing decision when organizing scheduled group buying activities}

Merchants organize booking group purchases while direct sales, and the success rate of group purchase is $\mu$. At this time, there are certain customers who choose to buy directly. At this time, the expected number of customers to participate is :

$$
\mu(1-\lambda)(A-B P)+(1-\mu)(A-B P)
$$

For other customers, the expected number of group purchases is: $\mu\left[\lambda(A-B P)+\theta B\left(P-P_{1}\right)\right]$

Therefore, the expected profit function of the merchant can be expressed as:

$$
\begin{aligned}
& \pi_{l}\left(P, P_{1}\right)=\mu(P-C)(1-\lambda)(A-B P)+ \\
& \mu\left(P_{1}-C\right)\left[\lambda(A-B P)+\theta B\left(P-P_{1}\right)\right] \\
& +(1-\mu)(P-C)(A-B P)
\end{aligned}
$$

Proposition 1:When $\theta$ and $\lambda$ is satisfied:

$$
0<\lambda<2 \sqrt{\frac{\theta}{\mu}}-\theta
$$

there are the optimal direct purchase price $P^{*}$ and the optimal reservation group purchase price $P_{1}^{*}$, satisfying:

$$
\begin{aligned}
& p^{*}=\frac{\left(2 \theta-\mu \theta \lambda-\mu \lambda^{2}\right) A+\left(2 \theta-\mu \theta \lambda-\mu \theta^{2}\right) B C}{B\left[4 \theta-\mu(\theta+\lambda)^{2}\right]} \\
& P^{*}{ }_{1}=\frac{(1-\mu \lambda)(\theta+\lambda) A+\left(3 \theta-\mu \theta \lambda-\mu \theta^{2}-\lambda\right) B C}{B\left[4 \theta-\mu(\theta+\lambda)^{2}\right]}
\end{aligned}
$$

Proof: The Hessen matrix of $P$ and $P_{1}$ obtained from the formula (6)、(7) $\pi_{1}\left(P, P_{1}\right)$ is

$$
\left(\begin{array}{cc}
-2 B(1-\mu \lambda) & -\mu B(\lambda-\theta) \\
-\mu B(\lambda-\theta) & -2 \mu \theta B
\end{array}\right)
$$

Because $-2 B(1-\mu \lambda)<0, \quad 0<\lambda<2 \sqrt{\frac{\theta}{\mu}}-\theta$ is established, the above Hessen is a negative definite matrix $\pi_{1}\left(P, P_{1}\right)$ is about $\left(P, P_{1}\right)$ the concave function. $\left(P^{*}, P_{1}^{*}\right)$, which is the optimal solution of $\max \pi_{1}\left(P, P_{1}\right)$ can be obtained by the first-order necessary conditions.

According to the obtained merchant profit function, the first-order partial derivatives of $P$ and $P_{l}$ are obtained respectively:

$$
\begin{aligned}
& \frac{\partial \pi_{l}\left(P, P_{1}\right)}{\partial P}=(1-\mu \lambda)(A-B P)-B(1-\mu \lambda)(P-C)+ \\
& \mu B\left(P_{1}-C\right)(\theta-\lambda) \\
& \frac{\partial \pi_{1}\left(P, P_{1}\right)}{\partial P_{1}}=\mu \lambda(A-B P)+\mu \theta B\left(P-P_{1}\right) \\
& -\mu \theta B\left(P_{I}-C\right)
\end{aligned}
$$

From $\frac{\partial \pi_{l}\left(P, P_{1}\right)}{\partial P}=0$ and $\frac{\partial \pi_{l}\left(P, P_{1}\right)}{\partial P_{1}}=0$ the only optimal solution is $P^{*}, P_{1}^{*}$ which satisfies the formula (6)、 (7).After calculation, the final total expected profit is expressed as:

$$
\pi_{l}\left(P, P_{1}\right)=\frac{\theta(1-\mu \lambda)(A-B C)^{2}}{B\left[4 \theta-\mu(\theta+\lambda)^{2}\right]}
$$

By comparing the price and profit when the merchant organizes a group purchase and when the product is sold directly, some conclusions can be drawn:

Conclusion 1: From a price point of view, it exists $P^{*}>P_{0}^{*}>P_{1}^{*}$ because:

$$
\begin{aligned}
& P^{*}-P_{0}^{*}=\frac{\mu\left(\theta^{2}-\lambda^{2}\right)(A-B C)}{2 B\left[4 \theta-\mu(\theta+\lambda)^{2}\right]} \\
& P_{0}^{*}-P_{1}^{*}=\frac{(\theta-\lambda)(2-\mu \theta-\mu \lambda)(A-B C)}{2 B\left[4 \theta-\mu(\theta+\lambda)^{2}\right]}
\end{aligned}
$$

From $\theta \geq \lambda, 0 \leq \lambda<1,0<\theta \leq 1$, we can get $P^{*}-P_{0}{ }^{*}>0$,

$P_{0}{ }^{*}-P_{1}^{*}>0$,so $P^{*}>P_{0}{ }^{*}>P_{1}^{*}$ is established, the general sales price when the group purchase is organized is higher than the retail price when the group purchase is not organized, and is higher than the reservation group purchase price set by the merchant. This shows that in order to attract additional purchasers, the merchants have increased the normal price after organizing the reservation group purchase and set the reservation group purchase price relatively low. When the booking group purchase price formulated by the merchant is lower, the market capacity can be expanded. Booking a group purchase will make non-participating consumers pay a higher price while allowing participants to enjoy a relatively low price. Part of the benefits of non-participating consumers will be transferred to the participants.

Conclusion 2: From the perspective of supplier profits, 


$$
\begin{aligned}
& \pi_{1}{ }^{*}>\pi_{0}{ }^{*} . \\
& \quad \text { Because } \pi_{1}{ }^{*}-\pi_{0}{ }^{*}=\frac{\mu(\theta-\lambda)^{2}(A-B C)^{2}}{4 B\left[4 \theta-\mu(\theta+\lambda)^{2}\right]}, \text { from }
\end{aligned}
$$

Proposition 1 and the assumptions, we know that $\pi_{1}{ }^{*}-\pi_{0}{ }^{*}>0$ Constant establishment, which means $\pi_{1}{ }^{*}>\pi_{0}{ }^{*}$ Constant establishment. This means that the profit of the merchants organizing the reservation group purchase is always greater than the direct sales, and it is more beneficial to carry out the reservation group purchase.

\section{Conclusion}

Under various assumptions, this article constructs a pricing model for online group-buying. Based on deduction and calculations, it finally concludes how merchants should adopt corresponding price-setting strategies and profit models in order to realize the healthy development of enterprises and maximize profits. Summarizing the main content of this article, the following conclusions are obtained:

(1) Under different booking group purchase success rates, various merchants should formulate different group purchase price strategies. In order to attract more customers after organizing the reservation group purchase, the merchant increased the normal price and set the reservation group purchase price relatively low. When the booking group purchase price set by the merchant is low, the market capacity can be expanded. Booking a group purchase makes the part of customers who did not participate in the group purchase pay a higher price, so that the customers who participated in the group get a lower price, and a part of the benefits of the customers who did not participate in the group were transferred to the party of the group.

(2) The profit when the merchant organizes a group purchase is always higher than the profit from direct sales. Lower booking group purchase prices will stimulate additional consumers to purchase goods and create conditions for merchants to enhance their brand value. At the same time, the profit of the merchant will also increase, and the increase in profit is directly proportional to the success rate of booking group purchases. Therefore, when merchants organize booking group purchases during direct sales, it is beneficial to attract additional consumers, increase sales, and win market share, which is more conducive to profit creation and better development of merchants.

(3) The price that users enjoy should be inversely proportional to the number of transactions. The more active the user is, the higher the transaction frequency and the number of times, the lower the price they enjoy. Moreover, consumers with a large number of transactions tend to have large consumption demands, and lower prices can stimulate them to conduct multiple transactions, mobilize the flow and transaction volume of the platform, and thereby increase the profit of the platform. If consumers like a certain business very much, the business volume of the business should be larger, and the business setting a lower price can reduce the churn rate and attract more consumers.

\section{References}

1. Webster J.r.,Yoram Wind.Organizational buying behavior,consumer behavior[J].New York: Holt, Rinehart and Winston,(04):238-246. (1968)

2. KauffmanR.J.,B.Wang.Building brand community[J].Journal of Marketing,(10):38-54.(2002)

3. Tang Fangcheng, Chi Kunpeng.Research on online group buying pricing strategy under bilateral network environment[J].China Management Science,(03): 185 -192.(2013)

4. LiGuohao, Chen Jingxian.Retailer group buying game with price and service competition at the same time[J].System Engineering,(11):13 -17.(2013)

5. Ju Chunhua, Wang Bei.A new online group buying model: active online group buying[J].Business Economics and Management, (09):9-14.(2006)

6. Chen Haitao, Li Tongqiang. A new model of online group buying based on the perspective of information sharing[J].Information Science,(08):79-82.(2015) 